\section{GENRE}

en séries

\section{Genre en séries}

Cinéma, télévision, médias

5 | 2017

Masculinités imag(in)les 2

\title{
So, you like show tunes? Jouer la gamme des masculinités dans Glee
}

\section{Fanny Beuré}

\section{(2) OpenEdition}

1 Journals

\section{Édition électronique}

URL : http://journals.openedition.org/ges/857

DOI : $10.4000 /$ ges.857

ISSN : 2431-6563

\section{Éditeur}

Presses universitaires de Bordeaux

\section{Référence électronique}

Fanny Beuré, "So, you like show tunes ? Jouer la gamme des masculinités dans Glee », Genre en séries [En ligne], 5 | 2017, mis en ligne le 01 juin 2017, consulté le 22 mars 2021. URL : http:// journals.openedition.org/ges/857 ; DOl : https://doi.org/10.4000/ges.857

Ce document a été généré automatiquement le 22 mars 2021.

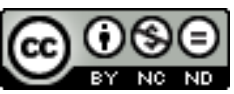

La revue Genre en séries est mise à disposition selon les termes de la Licence Creative Commons Attribution - Pas d'Utilisation Commerciale - Pas de Modification 4.0 International. 


\title{
So, you like show tunes? Jouer la gamme des masculinités dans Glee
}

\author{
Fanny Beuré
}

1 «Don't Stop believing. Hold on to that feeling... » : ces quelques phrases de la chanson du groupe Journey, reprise dès le pilote, résument le message optimiste et volontariste porté par la série musicale américaine Glee (Fox, 2009-2015). Relatant le quotidien d'une chorale lycéenne dans une petite ville fictive de l'Ohio, la série se situe à mi-chemin entre le backstage musical et le teen movie, et aborde des sujets variés, parfois très lourds (la mort d'un adolescent, la maladie, le harcèlement, le suicide), souvent plus légers (les rivalités entre élèves, la mode de la danse Twerk) et avec de fréquentes références aux enjeux sociétaux de l'Amérique contemporaine (droits des homosexuels, contrôle des armes à feu, crise économique). Cependant, tous les épisodes partagent le même thème conducteur: la quête identitaire et les incertitudes de l'adolescence, la difficulté ordinaire de s'accepter tel que l'on est.

2 Glee met effectivement sur le devant de la scène des personnages que leur couleur de peau, orientation sexuelle, identité de genre, handicap ou corpulence rendent sujets aux moqueries, mais qui parviennent finalement à s'affirmer. Parmi ces difficultés, les questions relatives au genre et à la sexualité tiennent une place de choix, qu'il s'agisse des relations entre filles et garçons, entre homosexuels et hétérosexuels, ou des questionnements sur la fluidité des identités de genre. Si la série a été saluée pour avoir ainsi rendu visibles de nombreuses minorités ${ }^{1}$, elle a également été critiquée pour son opportunisme et le caractère parfois simpliste de ses représentations (Rosenberg; Santori).

La variété des enjeux socioculturels abordés par la série n’a pas échappé à la recherche universitaire, en particulier anglo-saxonne (Johnson et Faill ; Parke). Cependant, peu d'analyses prennent en compte les spécificités du genre musical, c'est-à-dire l'emploi de numéros chantés et dansés pour faire avancer l'action et incarner les rapports de pouvoir entre les personnages. Par ailleurs, si une grande majorité d'articles se concentrent sur les personnages LGBTQ, ce n'est pas la seule brèche qu'elle ouvre dans les représentations traditionnelles des identités sexuelles et de genre. 
4 En effet, un double questionnement traverse Glee durant ses six saisons et fait écho à la nature hybride de la série. D'abord, en tant que série musicale, Glee s'interroge sur la place de la danse et de la musique dans l'expression des identités sexuelles et de genre. Une menace est particulièrement évoquée : le risque de dévirilisation encouru par les hommes qui se donnent en spectacle. Ensuite, en tant que série adolescente, Glee se demande ce que signifie grandir et comment le passage à l'âge adulte implique une conformation à certains rôles genrés. Si la série compte plusieurs personnages d'adolescentes, aucune ne semble s'interroger sur ce que signifie «devenir une femme » car leurs préoccupations concernent plutôt la carrière ou la sexualité - des problématiques auxquelles elles sont confrontées avant même le passage à l'âge adulte. En revanche, la préoccupation de "devenir un homme» est omniprésente pour les protagonistes masculins de la série, qu'elle soit directement exprimée par les adolescents angoissés par le passage à l'âge adulte ou que les questionnements de ces derniers renvoient les adultes à leurs propres interrogations sur leurs rôles et leurs responsabilités.

5 À travers l'analyse conjointe du récit et des numéros musicaux, cet article se propose d'étudier l'ampleur de la gamme des masculinités représentées dans Glee. Ces masculinités s'expriment à l'intérieur comme à l'extérieur du glee club, qu'elles soient homosexuelles ou hétérosexuelles, adolescentes ou adultes. Cependant, si la série paraît parfois repenser la place de la masculinité hégémonique, elle ne va généralement pas au bout de ses intentions, dans une constante négociation de ce qui peut être porté à l'écran ou non. Nous examinerons d'abord dans quelle mesure Glee renouvelle la représentation des masculinités dans la musique et la danse, "problème " classiquement posé par la comédie musicale. Nous interrogerons ensuite l'impératif récurent de "devenir un homme » en analysant comment se négocient les rapports de force entre différentes figures masculines, notamment entre les adolescents et leur père, souvent absent. Enfin, nous évoquerons les figures des entraîneuses/eurs Sue Sylvester (Jane Lynch) et Shannon/Sheldon Beiste (Dot Jones), dont les nombreux traits de caractère « virils » mettent au défi les masculinités de la série ${ }^{2}$.

\section{Rendre chanter et danser acceptables pour les hommes}

6 Parce qu'elle donne la primauté au spectacle plutôt qu'à l'action, la comédie musicale est traditionnellement envisagée comme un genre "féminin». Dès lors, la représentation des personnages masculins y pose souvent problème puisqu'elle contrevient à la répartition entre sujet et objet de regard traditionnellement pratiquée au cinéma ${ }^{3}$. Steven Cohan rappelle ainsi combien le corps masculin y est mis en spectacle, notamment dans la danse. Il analyse la masculinité de Fred Astaire pour expliquer comment le musical hollywoodien résout ce paradoxe en «féminisant " les hommes qui dansent, sans pour autant les réduire à un objet de contemplation passif ${ }^{4}$. Si Glee se trouve confronté au même "problème » que le musical classique, la série propose, plus encore, de faire de cette supposée menace de dévirilisation l'un de ses enjeux narratifs essentiels, en particulier pour les deux premières saisons. Elle offre ainsi un espace original au questionnement des rôles genrés, que le musical hollywoodien ne pouvait s'autoriser. 
7 En effet, dès l'épisode pilote (Pilot, S01xE01), Glee évoque la menace de «féminisation »-forcément infâmante-encourue par les hommes qui chantent et dansent. Ainsi, lorsque Finn Hudson (Cory Monteith), quarterback de l'équipe de football, rejoint le glee club sous la pression de l'enseignant Will Shuester (Matthew Morisson) - ce dernier déplorant précisément le manque de garçons au sein du club-, le jeune homme subit des moqueries des autres joueurs de l'équipe. Son ami Noah « Puck» Puckerman (Mark Salling), archétype du bad boy, le provoque par exemple en lui demandant s'il quitte l'équipe pour rejoindre « Homo Explosion ». En détournant ainsi le nom du glee club (New Directions), il exprime clairement le raisonnement hétérosexiste qui prévaut à Mc Kinley: les garçons ne sont pas autorisés à exprimer leur sensibilité artistique, car c'est révéler une partie émotive - donc faible - d'eux-mêmes. Exprimer cette sensibilité est une activité réservée aux filles et par conséquent, ceux qui pratiquent cette activité sont soupçonnés d'homosexualité selon le stéréotype péjoratif associant traditionnellement homosexualité masculine et traits de caractère culturellement codés comme « féminins ».

8 C'est précisément la décision d'aller à l'encontre de ces interdits, de refuser l'idée que certaines activités seraient inappropriées pour un homme, qui est le point de départ de la série. À l'issue de l'épisode pilote, Finn rétorque à Puck qu'il poursuivra les deux activités : le glee club, jugé inadéquat pour son genre, mais aussi le football - sport qui, parce qu'il permet d'exprimer la force, voire la brutalité, conviendrait davantage aux garçons. L'avenir du glee club est scellé dans la performance collective et entraînante de "Don't Stop Believing» de la fin du pilote : l'instant est tellement emblématique que la séquence est reprise à l'identique dans l'avant-dernier épisode de la série. Là est son acte fondateur: quand le jeune quarterback assume autant le plaisir qu'il tire à se donner en spectacle que celui qu'il prend à faire la démonstration de sa force ${ }^{5}$.

9 La compatibilité entre, d'une part, le fait de correspondre à un certain nombre d'attentes en matière de comportement masculin et, d'autre part, le fait d'aimer chanter et danser, est abondamment discutée tout au long des deux premières saisons. La série propose de résoudre cette supposée contradiction selon un double mouvement, interrogeant de fait différentes variations de la masculinité. Ainsi, dans Glee, certaines modalités du chant et de la danse peuvent être compatibles avec la masculinité hégémonique, tandis que d'autres ont vocation à être endossées par des masculinités dites « subordonnées ", notamment homosexuelles ${ }^{6}$.

10 La première manière dont Glee cherche à rendre le fait de chanter et danser compatible avec la masculinité hégémonique est de faire une analogie avec une autre activité dont la dimension virile ne fait aucun doute : le sport. Dans Acafellas (S01xE03), Will shuester indique à Finn et Puck que pour mieux danser, il leur faut relâcher les hanches, comme au baseball. Il leur demande alors, pour s'entraîner, de répéter le geste de balancer une batte. Ce geste - et surtout, l'assurance qu'il reste bien viril quoiqu'employé dans le cadre du glee club - les détend et ils répètent le mouvement en musique, à grands coups de "yeah", se topant les mains à la fin de l'enchaînement pour exprimer leur camaraderie virile. Dans Preggers (S01xE04), Finn suggère à Shuester que les membres de l'équipe tireraient profit d'un entraînement en danse, pour assouplir leurs mouvements. Lorsque le professeur vient exposer l'idée aux joueurs et qu'ils protestent, Shuester évoque plusieurs grands noms de sportifs qui étaient également des performers : Jim Brown, Dick Butkus, O. J. Simpson. Tous étaient des " pretty tough guys» (de «sacrés durs »), mais ont poursuivi de grandes carrières en tant que 
performers. Shuester tâche donc d'effacer le soupçon de « féminité » attaché au glee club en déplaçant le spectacle (et particulier la danse) du domaine artistique vers le domaine sportif, que ce soit en soulignant la proximité des gestes ou en évoquant quelques modèles de virilité indiscutable pratiquant les deux disciplines. Ce parallèle n'est pas nouveau et apporte même un écho troublant aux préoccupations exprimées, cinquante ans plus tôt, par Gene Kelly à propos du musical hollywoodien classique (Cohan, 2005). En effet, aucun danseur n'a plus que Kelly bâti sa persona autour de l'idée que la danse est une activité virile, proche du sport ${ }^{7}$. C'est cette conception viriliste de la danse, fruit d'une anxiété ancienne, que Glee perpétue.

11 La série cherche également à montrer que le chant et la danse peuvent conforter des traits de caractère envisagés comme typiques de ceux qui incarnent la masculinité hégémonique. C'est particulièrement le cas dans l'épisode Acafellas (S01xE03), lorsque Will Shuester fonde, avec d'autres professeurs et quelques élèves, un chœur masculin. Devant le succès inattendu du groupe, les hommes qui le composent prennent confiance en eux : la musique et le chant servent ici de vecteurs à l'affirmation d'une identité masculine forte, notamment face aux insécurités liées aux défis de la paternité ou de la vie conjugale. Surtout, la puissance tirée de leur performance est clairement liée à l'effet qu'elle provoque sur les femmes: c'est expressément pour séduire des femmes plus âgées que Puck rejoint le groupe, tandis que Shuester apprécie combien le fait d'appartenir à un boys band donne un second souffle à sa vie sexuelle conjugale. La nouvelle assurance éprouvée par les membres du groupe est notamment liée au répertoire choisi. Les trois chansons ${ }^{8}$ que les Acafellas interprètent appartiennent au registre New Jack Swing ${ }^{9}$, genre dominant de la musique populaire noire américaine chantée entre la fin des années 1980 et le début des années 1990. Ils renvoient donc à des figures de masculinité urbaine forte où la virilité se mesure à la capacité de dominer l'espace et conquérir les femmes, tout en gardant une attitude décontractée. Mais en même temps que le show paraît porter ce prétendu discours viriliste, il sème également les germes de sa remise en cause en soulignant que cette virilité retrouvée n'est que mascarade. Ainsi, la nouvelle ardeur sexuelle manifestée par la femme de Will n'est pas une façon de reconnaître le pouvoir séducteur d'un mari assumant son côté macho : elle souhaite être enceinte pour véritablement porter le bébé qu'elle prétend attendre depuis plusieurs semaines. Par ailleurs, à l'épisode suivant (Preggers, S01xE04), Finn affronte Puck en lui demandant en quoi l'arrivée de Kurt, membre du glee club, serait une menace pour leur virilité collective, lorsque Puck avait lui-même rejoint les Acafellas, soulignant par là le caractère arbitraire des jugements relatifs aux performances de genre.

12 Enfin, Glee tente également d'accorder le chant et la danse avec l'expression d'une masculinité hégémonique en déplaçant le spectre des masculinités hégémoniques acceptables : des modèles moins outrageusement virils, mais tout aussi dominants. À deux reprises, Will Shuester marche ainsi dans les pas de Fred Astaire, peut-être l'acteur qui a le mieux incarné, à l'écran, l'élégance de la danse au masculin. Deux routines d'Astaire sont ainsi recréées dans la série : «You're All the World to Me » de Royal Wedding (Stanley Donen, 1951) dans Girls (and Boys) On Film (S04xE15) et "Cheek to Cheek " de Top Hat (Mark Sandrich, 1935), dans Puppet Master (S05xE07). Dans ces deux séquences en noir et blanc, Shuester revêt le costume iconique du danseur hollywoodien (haut-de-forme, frac et canne) et parvient à capter une partie de la masculinité aristocratique qui le caractérise. Cette similitude réside cependant davantage dans l'habit que dans la danse, tant les mouvements de Morrison manquent 
du magnétisme aérien propre à Astaire. Un autre modèle de masculinité élégante, plus moderne, est celle incarnée par Mike Chang (Harry Shum Jr.), un des membres plus discrets du glee club. Lors de deux spectaculaires solos-le «Bubble Toes» de Jack Johnson (A Night of Neglect, S02xE17) ou le «Cool» de West Side Story (Asian F, S03xE03) toute la virtuosité du danseur est donnée à voir, en particulier dans la fluidité assurée des mouvements acrobatiques qu'il effectue. La recréation de «Cool» multiplie ainsi les sauts par rapport à la version originale, notamment en hybridant avec la danse jazz de l'œuvre de Robbins, des figures évoquant plutôt la danse hip-hop (saut à une main, mouvement de tractions, etc.). Il s'inscrit ainsi dans un modèle de masculinité urbaine qui allie sans complexe grâce et athlétisme.

Si Glee tâche donc, de plusieurs manières, de montrer que chanter et danser peut correspondre à un modèle de masculinité hégémonique, la série tient également un autre discours: il est acceptable pour les hommes homosexuels - incarnations de masculinités « dominées » - de chanter et danser de manière moins virile.

Glee reprend l'association entre homosexualité masculine et féminité, en particulier lors des premières saisons, principalement à travers le personnage de Kurt Hummel (Chris Colfer). Ce lien est tissé non seulement en attribuant au personnage de Kurt un certain nombre de traits culturellement envisagés comme "féminins» (tenues extravagantes, goût pour ce qui brille, langage précieux, physique fragile-Sue le surnomme "Porcelain»), mais surtout dans les numéros musicaux. En effet, au sein du glee club, les performances sont fréquemment sexuées: filles et garçons sont alors divisés en deux groupes, chacun se mesurant à l'autre en chansons ${ }^{10}$. Dans ce contexte, Kurt est constamment associé aux groupes féminins. Dans Theatricality (S01xE20) il est le seul garçon à participer au numéro "Bad Romance » de Lady Gaga. Dans Never Been Kissed (S02xE06), lorsque Shuester demande aux garçons et aux filles de former deux groupes, il rappelle immédiatement Kurt à l'ordre hétéronormé (« Kurt, dans l'équipe de garçons »), sans même avoir besoin de se retourner pour savoir que le jeune homme se dirigeait vers les filles. Dans The Power of Madonna (S01xE15), Kurt chante "What It feels like for a girl » avec les autres garçons des New Directions : il semble faire partie du groupe, d'autant qu'un travelling circulaire capte tout à tour chacun des interprètes, présentant les différentes figures de la masculinité adolescente. La pleine intégration de Kurt est cependant mise à mal lorsque, à l'issue du numéro, Finn reproche collectivement aux garçons leur attitude misogyne et que Kurt déclare l'approuver en tant que « fille honoraire ». Du fait de sa sexualité, Kurt trouble l'ordre genré qui dirige la partition des New Directions.

Ce trouble ne s'exprime pas seulement dans l'organisation de la troupe, mais aussi dans la distribution des chansons. Chris Colfer est doté d'une voix inhabituellement aiguë pour un homme : contreténor, il peut atteindre les mêmes notes que les mezzos ou les sopranos. Son aptitude à interpréter les voix aiguës est régulièrement mise à profit, notamment lorsqu'il chante avec d'autres garçons et qu'il se charge de la voix " féminine » - c'est par exemple le cas lors de son duo sur «Baby, It's Cold Outside » avec Blaine (Darren Criss) (A Very Glee Christmas, S02xE10). Le fait que son aptitude vocale brouille les assignations traditionnelles de genre est particulièrement mis en exergue lorsqu'il dispute avec Rachel (Lea Michele) le privilège de chanter "Defying Gravity », de la comédie musicale à succès Wicked (Wheels, S01xE09). Les paroles sont ici remarquablement consonantes avec l'enjeu narratif: défier les lois de la gravité, en avoir «assez d'accepter les limites dictées par quelqu'un d'autre ${ }^{11}$ signifie pour Kurt 
aller à l'encontre des stéréotypes de genre et se battre pour obtenir le droit d'interpréter un morceau traditionnellement chanté par une femme. Le nœud dramatique se resserre alors sur sa capacité à atteindre le «fa " aigu final, note d'autant plus difficile à atteindre que, dans cette "torch song ", elle doit être chantée en voix belt (voix de poitrine). Le montage alterné de la séquence fait apparaître combien Kurt maitrise la chanson aussi bien que Rachel, chacun redoublant d'intensité. Finalement, il échoue à dessein, par crainte que sa victoire ne sème trop de trouble dans les normes de genre (son père a reçu un appel malveillant l'insultant). Comme à l'ordinaire dans Glee, la musique métaphorise les problèmes auxquels sont confrontés les personnages : parvenir à chanter le «fa " aigu de la dernière phrase, serait pour Kurt l'occasion de pleinement s'affirmer et s'assumer tel qu'il est. Il a, plus tard dans la série, une nouvelle occasion de chanter «Defying Gravity» (100, S05xE12) : il ne manque alors aucune note, ce qui souligne le chemin parcouru.

Non seulement les performances de Kurt provoquent un trouble genré, mais elles invitent également à questionner la fluidité des identités de genre en mettant ostensiblement en scène leur performance. C'est par exemple le cas lorsque Kurt recrée «Le Jazz Hot!» de Victor Victoria (Duets, S02xE04), pour illustrer l'acceptation de ses côtés «féminin » et " masculin ». Dans ce numéro, Kurt porte un costume double : soie blanche à franges sur le côté droit, smoking noir sur le côté gauche - le couple noir/ blanc rejouant la dichotomie traditionnelle du masculin et du féminin. La chorégraphie reflète également la position ambiguë de Kurt par rapport aux stéréotypes de genre: s'il se mêle parfois aux lignes de danseurs en smoking qui l'entourent, il interprète le plus souvent une routine opposée à la leur: il fait face au public alors les autres danseurs sont filmés de dos et vice versa. À la fin, il est soulevé par les autres danseurs, figure évoquant les male chorus des musicals hollywoodiens, où la star féminine est représentée telle un bijou dans un écrin de boys qui l'adorent. En étant au centre des attentions masculines, Kurt embrasse un rôle "féminin", mais en n'étant véritablement ni garçon, ni fille, il incarne un personnage véritablement queer.

Enfin, il n'est pas innocent que deux des morceaux qui problématisent de façon aussi radicale cette question de l'inadéquation genrée - et, par suite, sexuelle - soient des classiques de comédie musicale. En effet, la série rejoue fortement une tradition d'adéquation entre le public gay masculin et la comédie musicale ${ }^{12}$. Sur les 57 chansons interprétées par Kurt dans la série ${ }^{13}, 21$ sont des chansons de comédies musicales, qu'il s'agisse de films musicaux hollywoodiens («Le Jazz Hot!» de Victor/Victoria ou «Come What May» de Moulin Rouge!), de standards de Broadway ("Mister Cellophane» de Chicago ou "Rose's Turn» de Gypsy) ou de chansons associées aux divas du musical (la recréation, avec Rachel, du medley "Get Happy/Happy Days Are Here Again» originellement interprété par Judy Garland et Barbra Streisand). S'il partage cette obsession avec Rachel, le cas de la jeune fille réitère également l'association entre les gays et le musical car son amour pour Broadway est régulièrement mis en lien avec le fait qu'elle a été élevée par un couple d'hommes.

18 Cependant, si le personnage de Kurt est celui qui apparait le plus tôt et est l'un des plus développés, il n'est pas le seul homme gay de la série. Glee présente également d'autres formes de masculinité homosexuelle, plus proches du modèle hégémonique, et dont la relation à celui-ci est peut-être plus complexe. C'est par exemple le cas du personnage de Blaine Anderson (Darren Criss), petit ami de Kurt. Tout en assumant pleinement son homosexualité, Blaine correspond par certains aspects à la masculinité hégémonique : 
il pratique le football, la boxe et aurait même fondé le fight club secret de Dalton Academy (Hold On to Sixteeen, S03E08). Lorsque Kurt promet à Rachel et Mercedes qu'ils assisteront au match des Titans, l'équipe de McKinley, car « ils aiment le football », il se reprend bien vite : «Enfin, Blaine aime le football. Moi, j'aime les foulards » (Preggers, S01xE04), suggérant par là qu'au sein de leur couple, les hobbies sont distingués selon les assignations traditionnelles du masculin et du féminin. Naturellement, dans Glee, l'aptitude de Blaine à incarner la masculinité hégémonique se traduit musicalement par sa capacité à être un male lead convaincant. Dans Blame It on the Alcohol (S05xE14), sa performance de "Don't You Want Me » avec Rachel le conduit même à questionner sa propre sexualité comme si, selon le schéma bien connu de la comédie musicale, l'alchimie démontrée dans la musique ne pouvait révéler autre chose qu'une attirance sexuelle. Il affiche en outre une élégance sobre et un peu désuète, qui n'est, là aussi, pas sans rappeler le charme suranné des musicals classiques. Cependant, si la masculinité portée par Blaine incarne certains traits de la masculinité hégémonique, elle demeure placée dans une nette relation de dépendance par rapport à celle-ci. Ainsi, lorsque Puck remercie tous les garçons - sauf Kurt - de lui avoir appris à "être un homme », il ajoute " même toi, Blaine ", suggérant que la sexualité de Blaine pourrait l'empêcher d'être un modèle de virilité (Choke, S03xE18). Sa position au sein des Warblers, chœur masculin de la fictive Dalton Academy, est également révélatrice de cette ambivalence. Chanteur vedette, puis coach, il détient une autorité qui s'inscrit dans l'acception traditionnelle de la virilité qui est soulignée par la mise en scène: il est, en quelque sorte, le chef reconnu et révéré d'un groupe de garçons en uniformes. Mais le hiatus surgit du fait que ce groupe est une chorale dont les membres sont de fait suspects d'homosexualité : Blaine lui-même considère comme une évidence que tous ses élèves sont gays (Homecoming, S06xE02).

19 Une autre figure de masculinité homosexuelle, plus virile encore, est celle de Spencer Porter (Marshall Williams). Ce personnage n'apparaît que dans quelques épisodes de la saison 6, mais le surnom que lui attribue Sue Sylvester, « postmodern gay kid (Loser Like $\mathrm{Me}$, S06xE01) résume bien les enjeux véhiculés dans la série par son apparition. Spencer fait partie de l'équipe de football et a un physique de dur à cuire. Lorsque Kurt cherche à le recruter pour faire partie du nouveau glee club qu'il dirige avec Rachel, Spencer le raille : l'association entre homosexualité et participation au glee club est dépassée. À présent, il est possible d'être gay tout en incarnant pleinement le modèle du mâle dominant qui intimide les autres élèves.

Enfin, la chanson de Beyoncé «Single Ladies ", interprétée à plusieurs reprises dans la série, nous a semblé révélatrice des variations proposées par Glee autour des masculinités. La première interprétation de "Single Ladies" advient au début de Preggers: dans sa chambre, Kurt danse en compagnie de Tina (Jenna Ushkowitz) et Britanny (Heather Morris). Le numéro est soigneusement mis en spectacle : il est filmé dans un élégant noir et blanc, Kurt porte un justaucorps à paillettes, Tina et Brittany, des bodys. Lors de cette première performance, plans moyens et plans de détail alternent dans un montage rythmé, forçant l'attention soit sur quelques mouvements iconiques de la chorégraphie (le geste de la main sur "put a ring on it») et sur la synchronie complice des trois interprètes. Dans cette distribution, il n'est d'ailleurs pas innocent que Kurt endosse le rôle de Beyoncé: non seulement il chante la voix principale, mais il est également placé au centre des deux danseuses - tout comme 
"Queen B» dans la chorégraphie originelle-effectuant parfois des mouvements opposés à elles.

Plus tard, c'est l'équipe de football de McKinley qui, sur le terrain, s'essaye à la célèbre chorégraphie. L'image de ces colosses se trémoussant en réclamant qu'on leur «passe la bague au doigt » est fortement incongrue et la séquence peut de toute évidence être considérée comme camp ou prompte à brouiller les normes de genre traditionnelles (Marcucci, 2013 : 78). Cependant, il nous semble que la façon dont la danse est filmée a ici vocation à la masculiniser et donc à minimiser le «risque» de troubler véritablement les normes de genre. Cette masculinisation intervient d'abord dans les costumes : les danseurs portent leur équipement complet de football américain, casque et coque compris, ce qui rend leur carrure encore plus imposante. La fonction de la danse compte également : il s'agit d'une danse offensive destinée à impressionner et dérouter l'adversaire, à la manière du haka des All Blacks. Par ailleurs, le montage, rapide et alterné, intègre de nombreux contre-champs sur ceux qui assistent au spectacle: les autres joueurs sont médusés, le coach Ken Tanaka répète certains des gestes de la chorégraphie, le public se lève et est emporté par le rythme. Le montage donne donc moins à voir la danse elle-même que les effets de celle-ci : en ce sens, l'équipe se soustrait au regard potentiellement réifiant du spectateur. Certains des mouvements les plus queer sont également légèrement mis en retrait: un raccord intervient lorsque les danseurs passent leur bras au-dessus de la tête puis avancent en faisant mine de se fouetter les fesses ; ils sont donc finalement de dos pour le dernier geste, ce qui rend celui-ci moins compromettant. Enfin, l'absence d'individualisation des danseurs minimise elle aussi le risque de réification. Alors que la chorégraphie originelle était un trio différenciant la star et ses danseuses, tous les Titans effectuent les mêmes gestes. Le cadrage et la mise en scène redoublent cette importance du groupe: les plans larges sont privilégiés, tandis que l'ouverture en quinconce de la danse (les danseurs se déhanchent d'abord par groupe de trois, puis se baissent avant de se relever et de se déhancher à nouveau tous ensemble) accroît d'autant la force de l'ensemble et l'impression de synchronisation du reste de la chorégraphie. Comme souvent dans le backstage musical, la réussite du numéro d'ensemble exprime la solidité des liens unissant les membres du groupe. Par la danse, ce sont donc les valeurs, finalement très sportives, de cohésion dans l'adversité et de solidarité offensive qui sont véhiculées.

La dernière interprétation de « Single Ladies » intervient plus tard dans la série, au cours de l'épisode Goodbye (S03xE22). Cette fois, c'est Burt Hummel (Mike O'Malley) qui endosse le rôle de Beyoncé, accompagné de Britanny et Tina. Il s'agit d'une allusion directe à la performance de Kurt dans sa chambre ; quelques images de la séquence en noir et blanc sont d'ailleurs intercalées avec la performance de Burt. Comme pour la reprise de "Defying Gravity ", la citation d'un précédent épisode a vocation à souligner l'évolution des personnages. En effectuant cette danse, qu'il avait autrefois regardée avec méfiance tant elle semblait peu virile pour être effectuée par un homme, Burt indique combien il a pleinement accepté la personnalité de son fils, sexualité et goûts musicaux compris. Plus encore, il joue lui-même sur la fluidité des identités de genre en enfilant un gant à paillettes pour l'occasion, tout en conservant son habit très «col bleu ", constitué d'une chemise à carreaux, d'un jean et d'une casquette. La prestation ne compromet cependant en rien la virilité de Burt puisqu'il est clair qu'elle a valeur d'hommage à Kurt. En se mettant à la place de son fils, mais en gardant visible la maladresse qu'il a à effectuer les pas, Burt indique sa bienveillance à l'égard de cette 
masculinité dominée sans pour autant s'y identifier. Néanmoins, les rapports de domination sont bien inversés le temps d'une chanson: pour une fois, c'est la masculinité de Burt qui est inadéquate, tandis que celle de Kurt est la référence.

Figure 1. « Single Ladies » par Kurt, Tina et Britanny

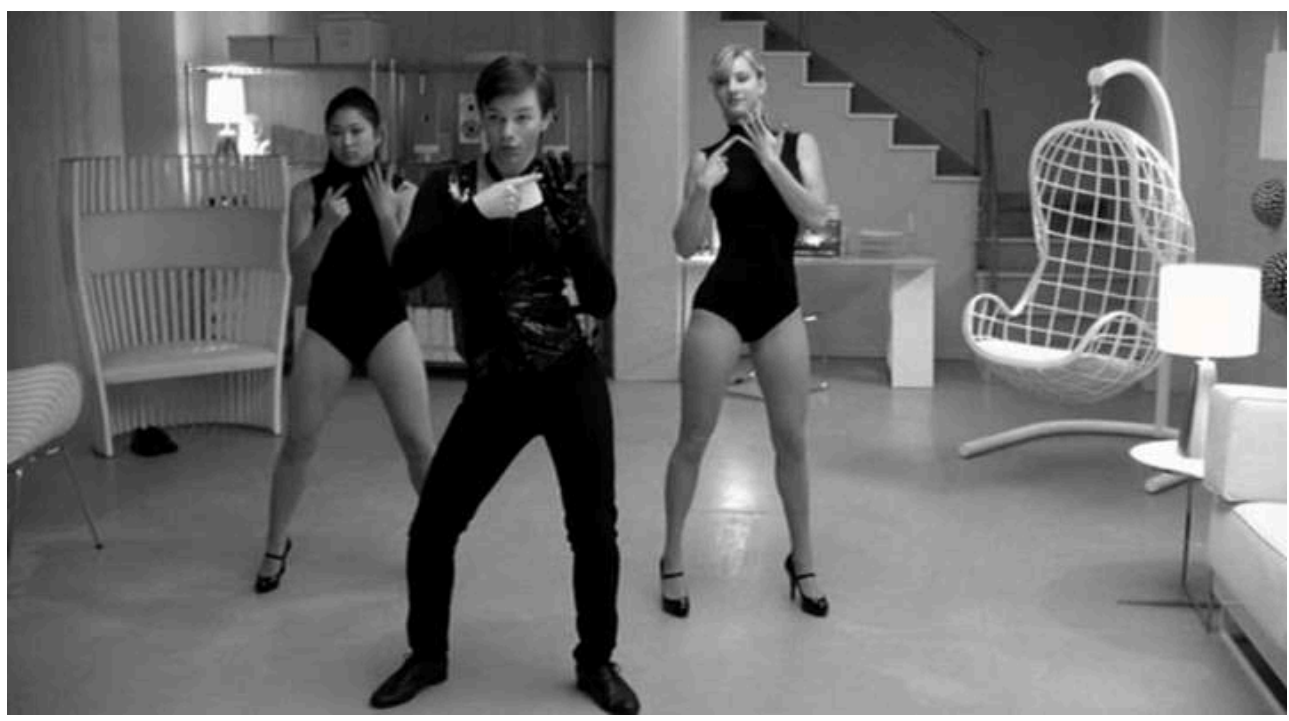

(Preggers, S01xE04)

Figure 2. « Single Ladies » par le Titans de McKinley

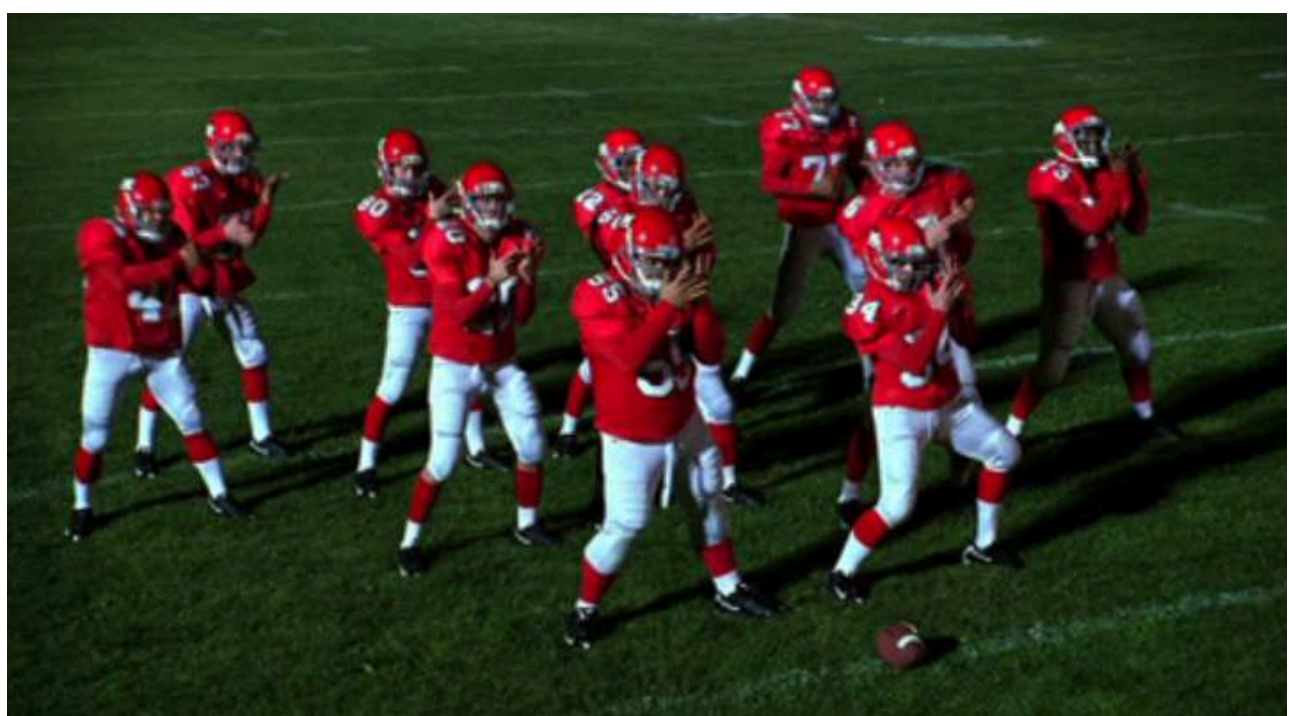

(Preggers, S01xE04) 
Figure 3. « Single Ladies » par Burt, Tina et Britanny

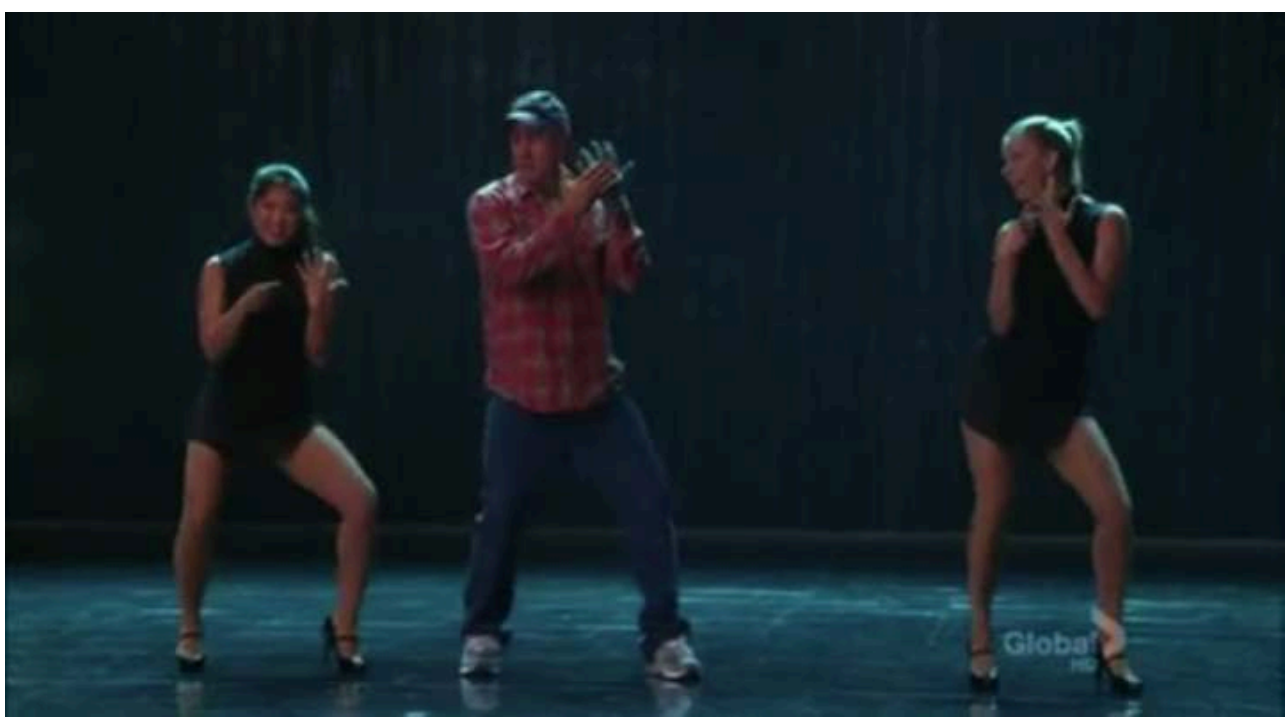

(Goodbye, S03xE22) selon Glee, hommes hétérosexuels et homosexuels se donnent en spectacle. Les performances des figures de masculinité hégémonique sont sans cesse "virilisées ». À l'inverse, avec le personnage de Kurt, Glee retourne de façon positive les stigmates habituels en montrant que ce n'est pas grave d'être gay et efféminé, à l'époque où de nombreux militants homosexuels préfèrent démontrer que les homosexuels ne sont pas efféminés. La limite à cette déclaration queer est qu'il n'est pas envisageable de présenter un personnage masculin qui soit à la fois hétérosexuel et efféminé. De plus, cette autorisation à l'expression «féminine " dans le chant et la danse est limitée à Kurt: les autres personnages gays restent bien, orientation sexuelle exclue, inscrits dans les canons de la virilité.

\section{Apprendre à devenir un homme}

Le deuxième enjeu longuement développé au fil de la série est le fait de « devenir un homme ». En effet, les rapports de domination entre les différentes masculinités, comme leur définition, sont en perpétuelle évolution ${ }^{14}$. Cet enjeu, "devenir un homme ", se pose pour la quasi-totalité des personnages masculins, adultes comme adolescents. D'abord, la nécessité de se trouver un modèle est une idée fixe chez la plupart des personnages. Finn est celui qui l'évoque le plus fréquemment, affirmant avoir puisé ses modèles auprès de sources variées, aussi bien auprès de Will shuester ${ }^{15}$, de sa mère ${ }^{16}$ que de Kurt $^{17}$. De façon presque obsessionnelle, Finn insiste sur le fait qu'ils ont remplacé la figure paternelle absente d'après laquelle le petit garçon était censé se modeler. La quête du père est d'ailleurs indiquée dès le premier épisode, et reliée aux enjeux musicaux de la série. Ainsi, un flashback sur son enfance fait surgir un souvenir musical et heureux : une chanson de Journey chantée avec le petit ami de sa mère. Ce moment d'harmonie dans une cellule familiale brièvement recomposée est placé sous le signe de la complicité virile, mais de manière si appuyée qu'il en devient immédiatement camp. Le jardinier séducteur aux cheveux longs brandit avec le petit garçon un jet phallique de peinture verte qui laisse assez peu de place au doute sur la

Genre en séries, 5 | 2017 
nature de la métaphore. C'est ce souvenir qui décide initialement Finn à rejoindre le glee club, et c'est encore cette image qui ressurgit, sur le terrain de football de McKinley, lorsque le jeune homme décide de ne pas se laisser impressionner par ses pairs et de demeurer dans le glee club. Dès le tout début de la série, la figure paternelle est donc associée à la musique, même si la virilité de cette figure est alors regardée de façon un peu moqueuse, à l'image du style musical qui l'identifie: un rock un peu ringard.

Cependant, si les déclarations de Finn sur ses modèles sont récurrentes, elles gardent toujours implicite ce que signifie vraiment être un homme. On n'est pas loin de la célébration d'un "éternel masculin ", comme si le fait d'" agir en homme " s'apprenait magiquement par contact. D'autres déclarations, un peu plus élaborées, nous éclairent davantage sur ce qu' " être un homme » signifie selon Glee. C'est particulièrement le cas de la déclaration que Will Shuester fait à Finn, au moment où il lui demande d'être témoin à son mariage (Yes/No, S03x10) :

Tu m'as appris ce qu'être un homme veut dire plus que quiconque. Tu défends tes amis. Tu n'as pas peur de pleurer et tu t'assureras que je ne ferai rien de stupide à mon enterrement de vie de garçon ${ }^{18}$.

26 Les traits énumérés par Shuester dépeignent une masculinité à la fois courageuse (défendre ses amis), sensible (ne pas craindre de montrer ses sentiments) et responsable. En outre, le fait que c'est l'enseignant adulte qui apprend de ses élèves adolescents ce que signifie être un homme indique combien cet apprentissage est en recomposition perpétuelle. Il ne s'agit donc pas tant de se conformer à un modèle que de constamment négocier avec les masculinités voisines. C'est la même idée qui soutient la déclaration de Burt à son fils (Dance With Somebody, S03xE17), alors que celui-ci s'apprête à quitter le lycée :

Kurt, vraiment, je suis si heureux et je suis si fier. Tu sais, toi et moi, on s'est appris

à être des hommes ${ }^{19}$.

Lorsque ces figures paternelles estiment être "devenus hommes » au contact d'un homme plus jeune, elles démontrent le caractère instable de cet être au monde masculin : non seulement on n'a jamais fini d'apprendre à « être un homme ", mais plus encore, tout le monde peut être à la fois élève et maître en la matière. D'ailleurs, ces figures paternelles se trouvent parfois confrontées à leur propre père. Dans Acafellas (S01xE03), le père de Will Shuester confie à son fils qui pense devenir père à son tour, qu'être un homme, c'est avant tout « avoir du cran »:

Être un père, bon Dieu, être un homme, c'est une affaire de tripes ${ }^{20}$.

28 Là encore, c'est le fils qui pousse presque automatiquement le père à devenir un homme : le courage dont Will fait preuve lorsqu'il s'agit de monter sur scène incite son père à reprendre ses études. Dans Glee, la relation au père est fondamentale dans l'affirmation de soi. Pour plusieurs personnages, l'interrogation sur le fait d'être un homme est d'ailleurs liée au fait d'être ou de devenir père : c'est le cas pour Finn et Puck au début de la première saison, pour Will et Burt tout au long de la série.

"Être un homme» est également lié à des problématiques sociales, par exemple lorsqu'il est question de réussite scolaire : dans Choke (S03xE18), les garçons du glee club aident Puck à réviser pour son examen final. Ce dernier les remercie «de lui avoir montré ce que cela signifie que d'être un homme ", ajoutant qu'il lui fut compliqué de grandir sans autre référent masculin à la maison que les joueurs de la NFL et les personnages de jeux vidéo. Apprendre à « être un homme » renvoie donc ici au fait de 
prendre son destin en main. De la même façon, le père de Sam déclare à son fils qu'il a dû « apprendre à être un homme beaucoup plus tôt que les autres garçons de son âge " car le déclassement familial l'a obligé à travailler pour subvenir aux besoins de la famille (Hold On to Sixteeen, S03xE08). Enfin, le "devenir homme» est également envisagé sous l'angle du handicap : Artie remercie ainsi ses camarades de l'avoir traité en adulte en lui faisant confiance pour la direction de West Side Story (The First Time, S03xE05). Il avoue que c'est à cette occasion qu'il s'est " pour la première fois de sa vie senti comme un homme adulte».

L'instabilité structurelle des masculinités se mesure aussi au fait que celles-ci évoluent fortement au fil du récit, de même que les rapports de force entre les différents hommes de Glee. La temporalité longue de la série - mais aussi la forme spécifique de la série adolescente et, plus encore, de la série en milieu scolaire - rend d'autant plus nécessaire l'évolution des personnages. À cet égard, le personnage de Kurt semble particulièrement emblématique: au fil des saisons, même si son homosexualité demeure une part importante de sa caractérisation, il évolue vers une forme de masculinité moins «dominée ». Ses tenues se font moins extravagantes et il n'hésite pas à faire la démonstration de sa force physique, par exemple en intervenant lors de l'agression d'un jeune homosexuel (Bash, S05xE15). Ce glissement est notamment lié à la transformation physique de Chris Colfer-l'un des plus jeunes interprètes-qui commence le tournage à 19 ans et perd peu à peu le visage enfantin qui lui avait valu d'être repéré. Il est aussi à mettre en relation avec une évolution morale du personnage qui s'accepte tel qu'il est. Cette affirmation morale et physique du personnage va de pair avec une modification du rapport de force qu'il entretient avec Blaine. Alors qu'au lycée Kurt était celui qu'il fallait protéger, Blaine se sent fragile une fois qu'il rejoint son compagnon à New York et qu'il ne trouve pas sa place dans la nouvelle vie de ce dernier. L'épisode No Sex in the City (S05xE16) évoque la modification de la relation de pouvoir entre les deux jeunes hommes; l'anxiété qui en résulte est exprimée dans le combat qu'ils se livrent pendant leur cours de maniement d'armes. Leur problème émotif explose dans une lutte virile mais se dénoue finalement dans la discussion : cette résolution en deux temps expose alors deux types de masculinité. Au combat violent viril mais qui ne résout rien - succède la forme adulte d'« être un homme » selon Glee: reconnaître ses torts et prendre ses responsabilités.

Enfin, la trajectoire de Finn nous semble particulièrement intéressante en tant que perpétuel exercice de redéfinition de la masculinité hégémonique. Si certains ont considéré que Finn proposait un modèle de masculinité défaillante (Brown, 2014), il nous semble au contraire que la trajectoire de Finn a pour vocation de réaffirmer le modèle dominant. Il apparait certes traversé de doutes et d'anxiété - en particulier par rapport à son avenir professionnel - mais il demeure, musicalement et narrativement, la figure centrale du glee club: male lead et courtisé par plusieurs filles. Les autres personnages masculins du club, chacun porteur d'un stigmate (racial, social ou physique) ne sont finalement que des faire-valoir pour la suprématie du jeune homme blanc. Par ailleurs, l'image que l'on retient finalement de Finn à sa mort est celle du quarterback, c'est-à-dire à la fois l'archétype du garçon populaire du lycée et, littéralement, le leader et le pilier (The Quarterback, S05xE03). 


\section{Les femmes viriles de Glee} Elle possède de multiples attributs socialement codés comme masculins: son nom évoque la bestialité (elle est " the beast »), elle exerce un métier d'homme (elle entraîne une équipe masculine), elle sait se montrer autoritaire et parler franchement (c'est elle qui parvient à raisonner Puck à la mort de Finn). Cette virilité passe également par des traits corporels, qu'il s'agisse du physique imposant de l'actrice Dot Jones, athlète haltérophile, ou de l'appétit hors du commun de son personnage, qui dévore régulièrement, à mains nues, plusieurs poulets rôtis. Pour autant, Shannon Beiste présente aussi une vulnérabilité culturellement envisagée comme typiquement féminine : elle recherche l'amour (Never Been Kissed, S02xE06), ne craint pas de pleurer en écoutant une chanson émouvante (The First Time, S03xE05) et est même victime de violences conjugales (Choke, S03xE18). Deux chansons du personnage sont d'ailleurs révélatrices de son positionnement ambivalent: "One Bourbon, One Scotch, One Beer» (Blame it on the Alcohol, S02xE14) et « Jolene » (I Kissed a Girl, S03xE07). La première, effectuée en duo avec Will dans un club de country est un numéro typique de camaraderie masculine, alors que la deuxième est une supplique destinée à la femme qui a séduit l'homme qu'elle pense perdre. Dans les deux cas, le style de la musique (respectivement le blues et la country) est connoté comme "masculin » et Dot Jones chante dans un registre grave, similaire à celui d'un ténor - la tonalité de "Jolene » étant une septième en dessous de la version originelle de Dolly Parton. Une fois de plus, c'est donc en musique comme dans le récit que Glee construit ses personnages : ici, une figure intéressante démontrant que la virilité n'est pas l'apanage des hommes et soulignant combien les caractéristiques assignées à chacun des deux sexes ne sont que le résultat d'une construction culturelle. Cependant, cette lecture du personnage est rendue caduque lorsqu'on considère Beiste comme un homme trans. Si cette transition peut être comprise comme une façon de réinterroger ce que c'est que devenir un homme, elle peut aussi être envisagée, de façon moins positive pour la série, comme une manière de "réaligner " son sexe avec des traits de caractère qui étaient trop discordants.

35 Une autre femme est fréquemment associée au masculin : l'entraîneuse des cheerleaders, Sue Sylvester. Elle l'est par son apparence, principalement par sa coiffure (elle porte les cheveux courts) et ses vêtements (elle est toujours vêtue du même survêtement, décliné en différentes couleurs). Certains de ses traits de caractère peuvent également être envisagés come « virils » : elle se montre violente (projette fréquemment les élèves 
contre leurs casiers, en plein couloir du lycée), méchante (ses insultes sont légendaires) et est prête à tout pour détruire le glee club. Surtout, Sue refuse les attributs "biologiques" de la féminité : elle dit ne pas avoir de menstruations ${ }^{22}$ et explique qu'elle ne veut pas d'enfant car elle n'a ni le temps, ni l'utérus pour en faire ${ }^{23}$. Mais l'incongruité de telles déclarations - qui vont d'ailleurs à l'encontre du récit puisque Sue aura un enfant - indique déjà le trait principal du personnage. Avec Sue, tout est exagéré, délirant, ironique : elle est véritablement camp. Ainsi, non contente d'être tous les jours en survêtement, elle décline même cet uniforme en robe de mariée: la récurrence du costume en fait un gag qui la rapproche finalement d'une caricature de super-héros. Dans Puppet Master (S05xE07), elle explique ainsi que le port du survêtement s'est avéré nécessaire pour se faire respecter. Une séquence en flashback qu'elle commente en voix-off, la montre parée d'une abondante chevelure et habillée d'un tailleur-jupe à dentelles roses typique des années 1980 ; cordiale avec les élèves, elle subit leur insolence et leurs moqueries. C'est alors qu'elle décide de se métamorphoser. Elle se coupe les cheveux et revêt le survêtement qui ne la quittera plus. L'effet est immédiat : les élèves fuient en la voyant, sur la musique de Carmina Burana, motif musical récurrent destiné à souligner de façon comique et disproportionnée la rage de Sue et la terreur qu'elle inspire. Le survêtement l'a donc parée, en quelque sorte, de superpouvoirs. Par ailleurs, elle est très régulièrement prise pour un homme $\mathrm{e}^{24}$ et adopte fréquemment des comportements inappropriés par rapport à son genre. La série développe cette inadéquation mais ne la condamne pas, d'autant que ce sont des moments qui la rendent attachante. En effet, lorsqu'elle s'aperçoit de ses failles, elle tente de les corriger, mais se rend compte qu'en essayant de correspondre aux impératifs esthétiques et comportementaux qui entourent la féminité, elle est en réalité plus artificielle et ridicule que jamais. Ces moments sont pour elle une façon de s'affirmer - alors même qu'elle est la première à railler les autres sur leur inadéquation genrée (féminité de Will Schuester, virilité de Beiste) et de se situer hors du genre, comme le note son fidèle lieutenant, la cheerio Becky Jackson (Lauren Potter) : «You have an allure that goes beyond gender 》 (Puppet Master, S05xE07).

Si Glee déploie sur les masculinités un discours foisonnant, la série n'est peut-être pas si ouverte à des modèles radicalement alternatifs. Certes, elle propose différents modèles d'homosexualité masculine, autorise les hommes hétérosexuels à danser et montre que les masculinités sont en perpétuelle construction. Néanmoins, la série est également révélatrice d'anxiétés persistantes.

D'abord, il n'y a guère que Kurt qui soit soustrait aux impératifs de virilité - et encore, ceci est moins vrai durant les deux dernières saisons. En outre, la série promeut une conception de la virilité extrêmement traditionnelle, synonyme de "prendre ses responsabilités »: même reconnaître ses faiblesses est présenté comme une force. Enfin, l'impératif de se trouver un modèle masculin - même si celui-ci est changeant et multiple - est révélateur d'un certain essentialisme. Ainsi, si Finn envisage de faire de Kurt un modèle de masculinité, c'est qu'il recherche partout des pères de substitution.

Alors que Shannon Beiste composait une figure, novatrice, de "femme virile", sa transition finale interroge. Si elle peut être considérée comme un moyen, salutaire, de rendre visible des personnages trans, elle peut également - dès lors que rien dans le récit ne la laissait présager - être perçue comme un réalignement du sexe avec le genre d'un personnage finalement trop éloigné des canons établis. La figure de Sue Sylvester est en définitive peut-être la seule qui interroge de façon pertinente la masculinité : 
c'est elle qui porte avec le plus d'aisance les attributs de la virilité, mais dans une posture camp soulignant la performativité des identités de genre.

\section{BIBLIOGRAPHIE}

BRown Sonya C. (2014), « Body Image, Gender, Social Class and Ethnicity on Glee», Studies in Popular Culture, 36.2 (Spring).

BUCHBINDER David (2008), « Enter the Schlemiel : The Emergence of Inadequate or Incompetent Masculinities in Recent Film and Television ", Canadian Review of American Studies, vol. 38/2, p. 227-245.

CLUM John M. (2001 [1999]), Something for the Boys : Musical Theater and Gay Culture, New York, St Martin's Press.

COHAN Steven (dir.) (2002) Hollywood Musicals : the Film Reader, London/New York, Routledge. COHAN Steven (2005), Incongruous Entertainment: Camp, Cultural Value, and the MGM Musical, Durham, Duke University Press.

CONNELL R. W. (1987), Gender and Power, Sydney, Allen and Unwin.

CONNELL R. W. et MESSERSCHMIDT James W. (2005), « Hegemonic Masculinity : Rethinking the Concept », Gender and Society, vol. 9/6, December.

FISHER Luchina (2011), « Born This Way’ Episode Has Sparks Flying. », ABCnews.com, 26 avril 2011, [en ligne] http://abcnews.go.com/Entertainment/glee-lady-gagas bornepisode/story? $\mathrm{id}=13451313$. [consulté le 28.09.2015].

JOHNSON Brian C. et FAILL Danielle K. (dir.) (2015), Glee and New Directions for Social Change, Rotterdam, Sense Publishers.

MARCUCCI Virginie (2013), « Glee et la reprise jubilatoire », TV/Series, $n^{\circ}$ 3, septembre.

MCCRACKEN Allison (2015), « Glee : The Countertenor and The Crooner », Antenna, 3 mai 2011, [en ligne] http://blog.commarts.wisc.edu/2011/05/03/glee-the-countertenor-and-the-crooner/. [consulté le 28.09.2015].

MULVEY Laura (1995), «Visual Pleasure and Narrative Cinema », Screen, vol. 16/3, p. 6-18.

PARKE Michelle (2014), Queer in the Choir Room - Essays on Gender and Sexuality in Glee, Jefferson, McFarland.

SANTORI Ann (2012), «Glee's disappointing treatment of social issues », The Examiner.com, 11 mai 2012, [en ligne] http://www.examiner.com/article/glee-s-disappointing-treatment-of-socialissues-1. [consulté le 28.09.15].

ROSENBERG Alyssa (2012), « Glee Is An Immoral Television Show And Its Time To Stop Watching It », Thinkprogress.org, 2 mai 2012, [en ligne] http://thinkprogress.org/alyssa/2012/05/02/475188/ glee-is-an-immoral-television-show-and-its-time-to-stop-watching-it/. [consulté le 28.09.2015]. 


\section{NOTES}

1. La série a été plusieurs fois récompensée lors des GLAAD Media Awards (récompenses décernées par la Gay \& Lesbian Alliance Against Defamation aux œuvres, médias et personnalités pour leur rôle dans la représentation de la communauté LGBT) et le critique conservateur Dan Gainor a même été jusqu'à accuser Ryan Murphy, créateur de la série, de promouvoir un " projet gay » (Fisher).

2. Alors que, pour désigner les caractéristiques spécifiquement féminines, on n'emploie que le terme de féminité, il existe pour les hommes plusieurs termes: virilité et masculinité. On entendra par virilité l'idéal abstrait qui définit ce que doit être un homme tandis que les masculinités, forcément plurielles, sont les façons réelles d'être un homme dans une société sexuée.

3. Laura Mulvey a montré combien le cinéma hollywoodien est patriarcal, dans sa forme comme dans son dispositif. En effet, les personnages féminins y sont des objets de désir passifs soumis à un triple regard masculin actif : celui de la caméra (le réalisateur), du personnage masculin et du spectateur. Laura Mulvey, "Visual Pleasure and Narrative Cinema », Screen, vol. 16/3, Fall 1995, p. 6-18.

4. Pour Cohan, c'est dans la narration et dans l'identité de ses personnages que la masculinité conventionnelle de Fred Astaire est affirmée avec force, tandis que le spectacle de son corps dansant, théâtralisé, repose sur trois dispositions considérées comme féminines : le narcissisme, l'exhibitionnisme et la mascarade. Steven Cohan (2002 [1993]), "'Feminizing' the Song-AndDance Man: Fred Astaire and the Spectacle of Masculinity in the Hollywood Musical ", dans Steven Cohan (dir.), Hollywood Musicals: The Film Reader, p. 87-101.

5. La décision de Finn contribue en outre à brouiller une autre frontière : celle entre les élèves populaires (dont font partie les jocks) et ceux qui ne le sont pas (les nerds que sont les New Directions, filles comme garçons). La revalorisation de la figure du nerd opérée par Glee fait en partie écho à ce qu'a observé David Buchbinder à propos du schlemiel, figure de masculinité inadéquate de plus en plus présente dans le cinéma américain contemporain. Pourtant, si Buchbinder voit dans le schlemiel un héros subvertissant l'ordre genré, il nous semble que la structure chorale de Glee contribue à réaffirmer cet ordre en opposant, au sein même du Glee club, des figures "traditionnelles» de nerds - dont le plus clair représentant est Artie (Kevin McHale), élève en situation de handicap moteur - à des modèles à la masculinité moins inadéquate. Voir David Buchbinder (2008), « Enter the Schlemiel : The Emergence of Inadequate or Incompetent Masculinities in Recent Film and Television », Canadian Review of American Studies, vol. 38/2, p. 227-245.

6. Nous empruntons à Raewyn Connell le terme de « masculinité hégémonique " pour désigner la déclinaison particulière de l'être au monde masculin qui domine à un moment donné et se fait passer pour l'unique modèle de comportement possible pour un homme. Cette masculinité hégémonique se construit en relation avec d'autres formes de masculinité, qu'elles soient « subordonnées ", « complices » ou «marginales ». Voir R. W. Connell (1987), Gender and Power, Sydney, Allen and Unwin.

7. Non seulement cette préoccupation a infusé l'ensemble de ses chorégraphies, elle a également parfois pris des allures de profession de foi, comme dans un épisode de l'émission Omnibus, au titre sans équivoque, «Dancing: A Man's Game ». Non content de danser aux côtés du boxeur Sugar Ray Robinson et de déclarer que la meilleure qualité du boxeur est le rythme, Kelly y met en parallèle des images de grands noms du sport (Mickey Mantle, Johnny Unitas) avec un danseur répétant son enchaînement, afin de souligner la proximité des gestes des sportifs et du danseur.

8. "This Is How We Do It» de Montell Jordan, «Poison", de Bell Biv DeVoe et «I Wanna Sex You Up », de Color Me Badd. 
9. Le New Jack Swing est un genre musical mêlant R\&B et hip-hop, caractérisé par une caisse claire très marquée et des voix aux accents soul.

10. C'est notamment le cas dans les épisodes The Power of Madonna (S01xE15), Never Been Kissed (S02xE06) et Girls (and Boys) On Film (S04xE15).

11. "I'm through accepting limits 'cause someone says they're so".

12. Sur le lieu commun de l'association entre les hommes gay et la comédie musicale, voir notamment : Steven Cohan, Incongruous Entertainment et John Mc Clum, Something for the Boys.

13. Sur l'ensemble des six saisons de la série, on dénombre ainsi 57 chansons dans lesquelles Kurt chante en solo, en duo, ou en groupe avec une part importante de phrases solo. Dans ce décompte, sont donc exclues celles où il chante au sein d'un ensemble.

14. C'est d'ailleurs précisément ce que précise Connell lorsqu'elle revient sur la définition de l'hégémonie. Celle-ci est un modèle qui se construit en actes, son caractère dominant évolue selon le temps et les contextes géographiques. Voir R. W. Connell et James W. Messerschmidt (2005), « Hegemonic Masculinity : Rethinking the Concept », Gender and Society, vol. 19/6, p. 832.

15. "I didn't have a father. Someone I could look up to. Model myself after. Someone who could show me what it really meant to be a man ». (Journey, S01xE22)

16. "Somehow even without one in the house, you taught me what it means to be a man". (Furt, S02xE08)

17. "Quite frankly, no one else has shown me as much as you about what it means to be a man ». (Furt, S02xE08)

18. "You told me more about being a man than anyone I've ever known. You stand up for your friends. You are not afraid to cry and you'll make sure I don't do anything stupid on my bachelor party ».

19. «Kurt, really, I am so happy and I am so proud. You know, you and me, we, uh, we made each other men».

20. «Being a father, hell, being a man, is all about guts".

21. Son personnage est introduit au début de la deuxième saison (Audition, S02xE01); elle remplace le coach Ken Tanaka.

22. "Sue: Iron table t? Keeps your strength up when you're menstruating./ Will : I don't menstruate./ Sue: Yeah? Neither do I. " (Showmance, S01xE02).

23. "Never wanted kids, never has the time, never had the uterus " (Vitamin D, S01xE06).

24. C'est le cas dans Opening Night (S05xE17) et dans Puppet Master (S05xE07).

\section{RÉSUMÉS}

À travers l'analyse conjointe du récit et des numéros musicaux, cet article étudie l'ampleur de la gamme des masculinités qui s'expriment dans la série Glee (Fox, 2009-2015). Différentes figures y sont développées, à l'intérieur ou à l'extérieur du glee club, homosexuelles ou hétérosexuelles, adolescentes ou adultes. Cependant, si la série paraît parfois repenser la place de la masculinité hégémonique, elle n'est peut-être pas tant ouverte aux modèles alternatifs. Glee renouvelle la représentation des masculinités dans la musique et la danse - «problème » classiquement posé par la comédie musicale - et discute les correspondances traditionnelles entre préférences musicales et sexuelles. La série interroge également l'impératif de "devenir un homme », en montrant comment les rapports de force entre différentes figures masculines se négocient au fil du temps, en particulier entre les adolescents et leurs pères. Néanmoins, les cas les plus 
intéressants sont peut-être les femmes «viriles» qui mettent véritablement au défi les masculinités de la série.

This article examines how a wide range of masculinities are expressed in the musical series Glee (Fox, 2009-2015), through the joint analysis of narrative and numbers. Several figures are indeed developed, inside or outside of the glee club, homosexual and heterosexual, teenagers or adults. If the series sometimes seems to rethink hegemonic masculinity, it may not be so open to alternative models. The series renews the representation of masculinity in music and dance "problem» that the classical musical already faced-and discusses the traditional correspondences between musical and sexual preferences. It also questions the duty to « become a man ", showing how the balance of power between different male figures are negotiated over time, particularly between adolescent boys and their fathers. However, the most interesting cases are perhaps Glee's « manly » women, who are genuinely challenging masculinities.

\section{INDEX}

Mots-clés : comédie musicale, cultural studies, danse, gender studies, musique, rapports sociaux de sexe

Keywords : cultural studies, dance, gender studies, gender relations, music, musical

\section{AUTEUR}

\section{FANNY BEURÉ}

Fanny Beuré est Docteure en Études Cinématographiques de l'Université Paris-Diderot -Paris 7. Elle a soutenu, sous la direction de Jacqueline Nacache, une thèse intitulée : « Let's Face the Music and Dance : la comédie musicale hollywoodienne classique au prisme de l'entertainment ». Outre la comédie musicale, ses domaines de recherche comprennent les analyses socioculturelles des contenus médiatiques (gender, gay et cultural studies notamment), la sociologie des publics et l'économie du cinéma. 